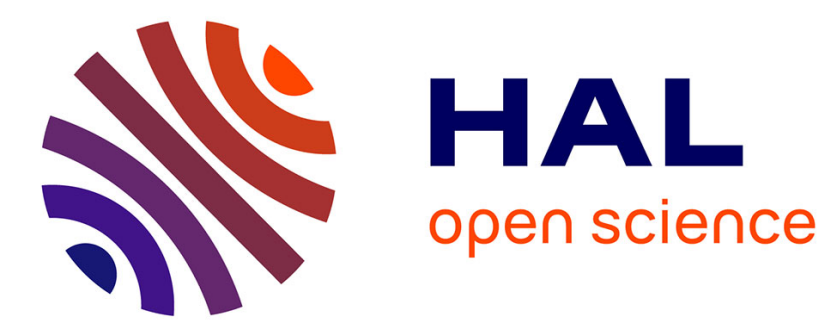

\title{
Trends in the cohesive properties of sp bonded elements
}

\author{
G. Allan, M. Lannoo
}

\section{To cite this version:}

G. Allan, M. Lannoo. Trends in the cohesive properties of sp bonded elements. Journal de Physique, 1983, 44 (12), pp.1355-1363. 10.1051/jphys:0198300440120135500 . jpa-00209723

\section{HAL Id: jpa-00209723 https://hal.science/jpa-00209723}

Submitted on 1 Jan 1983

HAL is a multi-disciplinary open access archive for the deposit and dissemination of scientific research documents, whether they are published or not. The documents may come from teaching and research institutions in France or abroad, or from public or private research centers.
L'archive ouverte pluridisciplinaire HAL, est destinée au dépôt et à la diffusion de documents scientifiques de niveau recherche, publiés ou non, émanant des établissements d'enseignement et de recherche français ou étrangers, des laboratoires publics ou privés. 
Classification

Physics Abstracts

$61.50 \mathrm{~L}$

\title{
Trends in the cohesive properties of sp bonded elements
}

\author{
G. Allan and M. Lannoo \\ Laboratoire de Physique des Solides, L.A. 253, ISEN, 3, rue F. Baës, 59046 Lille Cedex, France
}

(Reçu le 10 mai 1983, accepté le 12 août 1983)

\begin{abstract}
Résumé. - Les tendances concernant l'énergie de cohésion, la distance interatomique et la compressibilité des éléments à liaisons sp sont discutées dans le cadre d'une approche semi-empirique de liaisons fortes. On montre d'abord que deux modèles très simples permettent de décrire ces tendances ainsi que de démontrer la règle de Grimm-Sommerfeld pour les éléments dont la couche externe est plus qu'à moitié pleine. Un calcul numérique plus complet confirme ensuite ces prédictions, donnant des résultats en accord quantitatif avec les valeurs observées.

Abstract. - The trends in the cohesive energy, nearest-neighbour distance and compressibility of all sp bonded elements are discussed using a semiempirical tight binding approach. It is first shown that two very simple models describe these trends and also demonstrate the Grimm-Sommerfeld rule for elements with more than a half filled valence shell. A more complete numerical calculation is then performed which confirms these predictions, giving results in quantitative agreement with the observed values.
\end{abstract}

\section{Introduction.}

The cohesive properties of the sp bonded elements present systematic trends as a function of the number of electrons $N_{\mathrm{e}}$ in the sp band. As shown on figure 1 the cohesive energy is practically zero for $N_{\mathrm{e}}=0$ and 8 , i.e. for rare gases, and increases in a roughly symmetrical way to take its maximum value for $N_{\mathrm{e}}=4$. This is true for the first four rows of the periodic table. The nearest-neighbour distance on

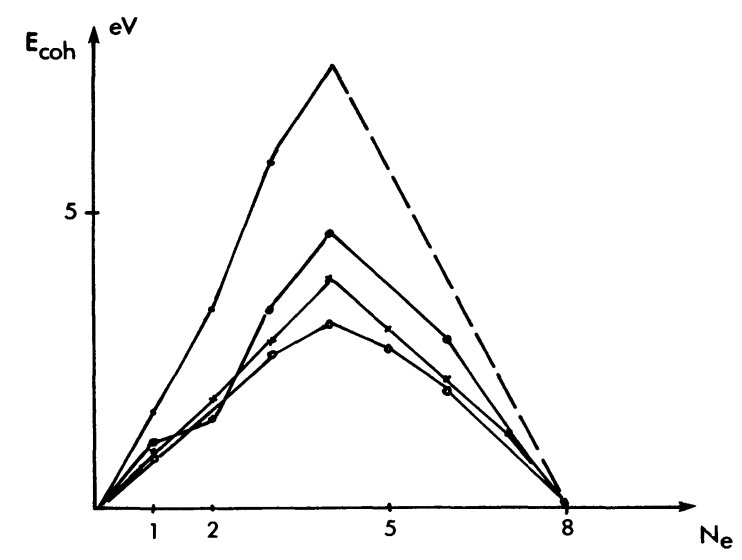

Fig. 1. - Experimental trends in the cohesive energy $E_{\mathrm{c}}$ versus the number of electrons $N_{\mathrm{e}}$ in the sp band, for the four first rows of the periodic table. Values are taken from [1]. the other hand falls regularly for $N_{\mathrm{e}}$ varying from zero to four and then remains roughly constant (Fig. 2). Finally the variation of the compressibility follows that of the cohesive energy [3].

This situation is similar in many respects to what happens in the transition metal series versus the filling of the $\mathrm{d}$ band. This was discussed some time ago in a tight binding description [4] and using the most simple version of the method of moments [5]. These studies resulted in a very nice and simple description

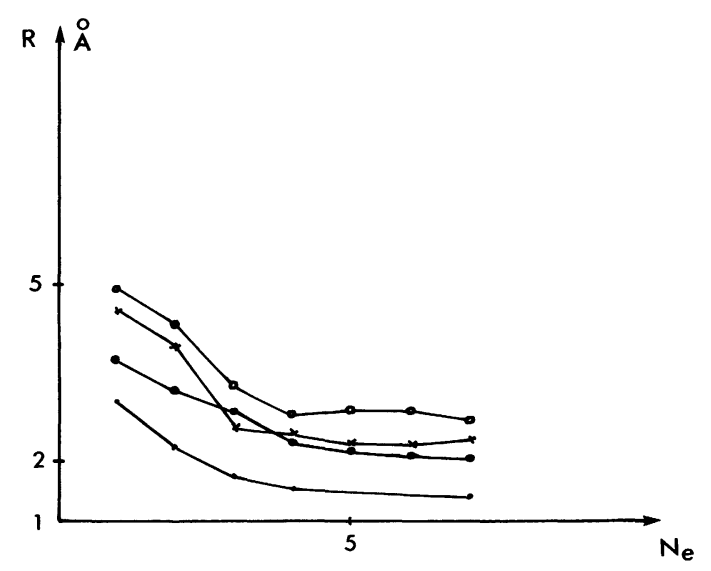

Fig. 2. - Experimental trends in the nearest neighbour distance $R$ versus $N_{\mathrm{e}}$. Values are taken from [2]. 
of the tendencies of the cohesive properties for that class of systems. Owing to these convincing results J. Friedel [6] attempted a tight binding determination of the trends in the cohesive energy of the sp bonded systems. Assuming that the variation of the total energy follows that of the sum of one electron energies he was able to predict the correct shape of this quantity versus $N_{\mathrm{e}}$. However this model was not complete enough to give predictions for the bond length as well as the compressibility.

In this work we want to extend the tight-binding description to the prediction of these last two quantities. For this we add to the sum of one-electron energies a repulsive contribution consisting of sums of pair potentials of the Born-Mayer type. With very few assumptions as regards the parameters of the model we show that the correct cohesive trends are easily reproduced but we are also able to demonstrate the Grimm-Sommerfeld or 8- $N_{\mathrm{e}}$ rule [7] for elements with $N_{\mathrm{e}} \geqslant 4$. We first do this in simplified analytic models which describe simply the basic physics of the problem. We use two opposite descriptions : one based on a method of moments, the other on the limit of pure covalency. The results are expressed in the form of general curves in reduced units valid for any row of the periodic table. The second part of the work consists in a complete numerical study of the cohesive properties of all possible simple structures as a function of $N_{\mathrm{e}}$. It confirms the predictions of the analytic models and allows a comparison of the relative stability of the different structures. Quantitative agreement is obtained as regards the trends versus $N_{\mathrm{e}}$. The main significative differences in the stability of various structures are also correctly predicted.

It is important to comment upon the motivations of this work compared to available calculations of the cohesive properties. It is clear that much better accuracy has been obtained in recent pseudopotential plus local density calculations [8]. However the advantages of the description which is proposed here are the following :

i) it gives a simple physical insight into the nature of the bonding and its relation with $N_{\mathrm{e}}$;

ii) it can be extended without too much trouble to disordered or amorphous systems ;

iii) it is ideally suited for the study of the properties of alloys and among these the important class discussed by E. Parthe [7] characterized by covalent (or partly ionic) bonds and coordination number smaller or equal to four.

\section{Description of the tight binding model}

We make use of a nearest-neighbour approximation (except for b.c.c. systems where, as we shall see later, we have used a second nearest-neighbour treatment). This approximation is characterized by intraatomic terms of the Hamiltonian $H$ which we write as

$$
\begin{aligned}
& E_{\mathrm{s}}=\langle\mathrm{s}|H| \mathrm{s}\rangle \\
& E_{\mathrm{p}}=\langle\mathrm{p}|H| \mathrm{p}\rangle
\end{aligned}
$$

where $s$ and $p$ are the corresponding atomic states. The interatomic matrix elements are written

$$
\beta_{i j}=\langle i|H| j\rangle .
$$

If we adopt a two-centre approximation, there will be only four independent $\beta$ parameters which are $\beta_{\mathrm{ss}}$, $\beta_{\mathrm{s} \sigma}, \beta_{\sigma \sigma}$ and $\beta_{\pi \pi}$ in Slater and Koster's notation [9]. The problem is to find simple prescriptions for the determination of these parameters. This was discussed by Harrisson [10] whose proposal is to use free atom values for $E_{\mathrm{s}}$ and $E_{\mathrm{p}}$ and a law in $\eta_{i j} / R^{2}$ for the $\beta_{i j}$ as functions of the interatomic distance $R$ (where the $\eta_{i j}$ are constants for a given class of materials).

In this work we shall adopt similar prescriptions for $E_{\mathrm{s}}$ and $E_{\mathrm{p}}$ (free atom values) and will write $\beta_{i j}$ under the form

$$
\beta_{i j}=\beta_{i j}^{0} \exp (-q R)
$$

where the $\beta_{i j}^{0}$ will take constant values for a given row of the periodic table. We also assume that the values between different rows are simply scaled by one constant factor (detailed values will be given later). The prescriptions for $q$ will also be described later. It is then possible from (3) to calculate the sum of one electron energies (or band structure energy) $E_{\mathrm{A}}$ for any given crystalline structure. With our assumptions $E_{\mathrm{A}}$ will be a function of $E_{\mathrm{s}}, E_{\mathrm{p}}$ and also of the $\beta_{i j}^{0}$ and $q R$.

Usually $E_{\mathrm{A}}$ is attractive, i.e. one gains energy by decreasing $R$ since the dominant $\beta_{i j}^{0}$ are negative. However to get the total energy one must add to $E_{\mathrm{A}}$, terms accounting for the following interactions which are omitted in $E_{\mathrm{A}}:$ i) attractive shifts in diagonal matrix elements due to the potential of neighbouring atoms, ii) repulsive electron-electron terms which are counted twice and iii) repulsive nuclei-nuclei interactions. For elemental crystals these three terms can be combined together as a sum of interactions between neutral atoms [11] which is repulsive and short ranged in nature. To this one must still add a correction due to the non orthogonality of the basis states which again is repulsive and short ranged [12]. In the following we simulate these repulsive terms by pair potentials of the Born-Mayer type truncated to nearest-neighbours (except for the numerical calculations on b.c.c. crystals). Then the repulsive energy per atom will be written :

$$
E_{\mathrm{R}}=\frac{Z}{2} C_{0} \exp (-p R)
$$

where $Z$ is the coordination number (number of nearest-neighbours). 
In order to understand qualitatively what happens we shall first apply simplifying techniques which will clearly indicate the physical origin of the trends. We first assume that the $\beta_{i j}^{0}, C_{0}, p, q$ are constant along a given row of the periodic table and that the essential effect on the cohesive energy $E_{c}$ and equilibrium interatomic distance $R_{\mathrm{e}}$ is due to the filling of the sp band. For the approximate calculations to be described first we shall have to consider two different situations for $N_{\mathrm{e}}<4$ or $N_{\mathrm{e}} \geqslant 4$. In the former case one has metallic behaviour corresponding essentially to a partially filled broad band. In the latter case there is tendency towards covalent bonding with formation of subbands of strong molecular or atomic character. We shall apply different analytical approximations to these two different situations.

\section{The metallic case $N_{\mathrm{e}}<4$.}

To simplify we neglect the difference $E_{\mathrm{p}}-E_{\mathrm{s}}$ and calculate the energy $E_{\mathrm{A}}$ using a simplified description of the density of states. We take a constant density of states over the whole bandwidth. In such a model the number of electrons $N_{\mathrm{e}}$ per atom is a linear function of the Fermi level position $E_{\mathrm{F}}$ while $E_{\mathrm{A}}$ is quadratic in $E_{\mathrm{F}}$ (see [4] for the same model applied to transition metals). This allows $E_{\mathrm{A}}$ to be expressed in the form (the atomic level is taken as the origin of energies).

$$
E_{\mathrm{A}}=-E_{\mathrm{AM}}^{0} \frac{N_{\mathrm{e}}\left(8-N_{\mathrm{e}}\right)}{16} \exp (-q R)
$$

where the quantity $E_{\mathrm{AM}}^{0}$ is proportional to some combination of the $\beta_{i j}^{0}$ which depends of the structure. For a given structure it will thus be a constant.

We can now add to (5) the repulsive part $E_{\mathrm{R}}$, which for fixed $Z$, can be written (see (4))

$$
E_{\mathrm{R}}=E_{\mathrm{R}}^{0} \exp (-p R) \text {. }
$$

The total energy per atom is the sum of $E_{\mathrm{R}}$ and $E_{\mathrm{A}}$. It can be minimized with respect to $R$ giving the equilibrium distance $R_{\mathrm{e}}$ the form

$$
R_{\mathrm{e}}=R_{\mathrm{m}}-\frac{1}{p-q} \ln \frac{N_{\mathrm{e}}\left(8-N_{\mathrm{e}}\right)}{16}
$$

with

$$
R_{\mathrm{m}}=\frac{1}{p-q} \ln \left(\frac{p E_{\mathrm{R}}^{0}}{q E_{\mathrm{A}}^{0}}\right) .
$$

The cohesive energy per atom $E_{\mathrm{c}}$ can be obtained as - $\left(E_{\mathrm{A}}+E_{\mathrm{R}}\right)$ evaluated at $R=R_{\mathrm{e}}$. This gives

$$
E_{\mathrm{c}}=E_{\mathrm{cM}}\left[\frac{N_{\mathrm{e}}\left(8-N_{\mathrm{e}}\right)}{16}\right]^{\frac{p}{p-q}} \text {. }
$$

The functions $R_{\mathrm{e}}\left(N_{\mathrm{e}}\right)$ and $E_{\mathrm{c}}\left(N_{\mathrm{e}}\right)$ are plotted in figure 3 . The experimental trends of figures 1 and 2 are clearly reproduced as was the case in the transition metal series (note that we take $p>q$ since it is generally
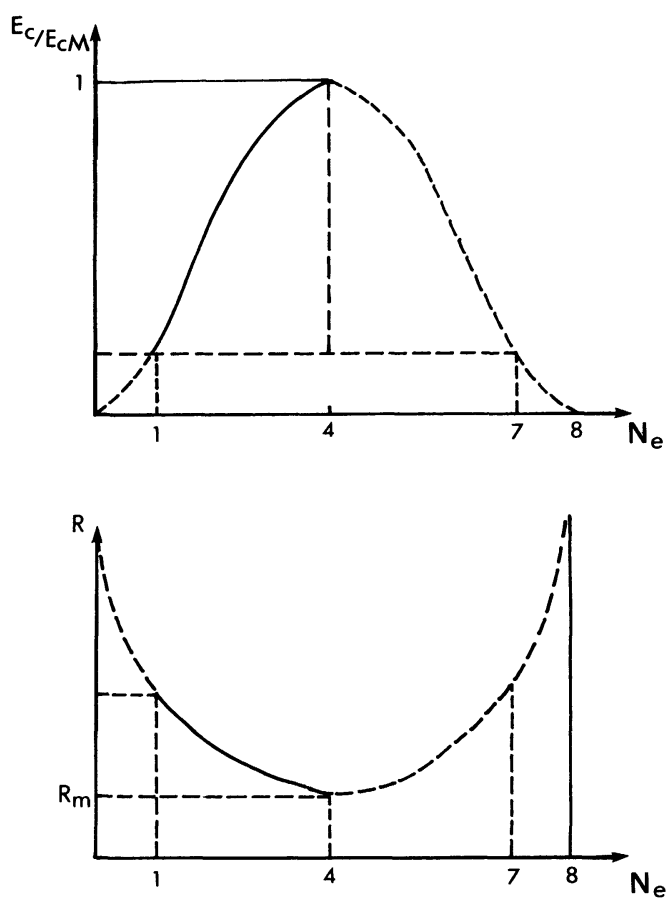

Fig. 3. - Trends in $E_{\mathrm{c}}$ and $R$ versus $N_{\mathrm{e}}$ deduced from a rectangular density of states, with $E_{\mathrm{s}}=E_{\mathrm{p}}$.

expected that the repulsive part is shorter ranged). One can also wonder what should be the influence of the coordination number $Z$. If the width of the constant density of states is calculated by a method of moments [5], then the quantity $E_{\mathrm{AM}}^{0}$ varies as $\sqrt{Z}$ while, as follows from (4) $E_{\mathrm{R}}^{0}$ is proportional to $Z$. It is easy to show that $E_{\mathrm{c}}$ behaves as

$$
E_{\mathrm{c}} \propto Z^{\frac{p-2 q}{2(p-q)}} .
$$

We shall see later that $p \sim 2 q$. Therefore the dependence of $E_{\mathrm{c}}$ on $Z$ is weak. This simplified model being only of qualitative value we shall discuss this dependence only for the numerical calculations of $E_{\mathrm{c}}$.

A final important feature concerning the behaviour of $E_{\mathrm{c}}$ and $R_{\mathrm{e}}$ can also be deduced in the same model. If we consider the limiting case where $E_{\mathrm{p}}-E_{\mathrm{s}}$ is large, the same calculation can be applied to the $s$ and $\mathrm{p}$ bands separately. In this limit the qualitative behaviour is pictured on figure 4 . The important conse-
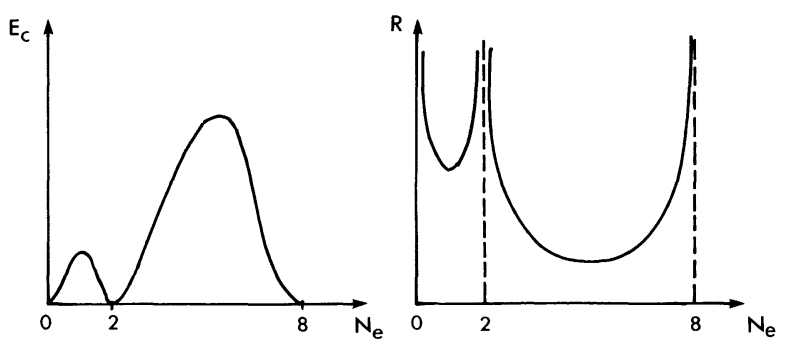

Fig. 4. - Trends in $E_{\mathrm{c}}$ and $R$ versus $N_{\mathrm{e}}$ for separated $\mathrm{s}$ and $\mathrm{p}$ bands. 
quence is that for $N_{\mathrm{e}}=2$ the cohesive energy vanishes and $R_{\mathrm{e}}$ tends to infinity. For this critical value of $N_{\mathrm{e}}$, the predicted results will be extremely sensitive to the precise value taken by $E_{\mathrm{p}}-E_{\mathrm{s}}$ as will be the case in the numerical calculation.

\section{The covalent case $N_{\mathrm{e}} \geqslant 4$ and the Grimm-Sommer- feld rule.}

In this section we derive another analytic approximation appropriate to covalent systems. This will allow us to demonstrate in closed form the Grimm-Sommerfeld rule [7] or $8-N_{\mathrm{e}}$ rule which states that

$$
Z=8-N_{\mathrm{e}} \text {. }
$$

It is known that such systems are characterized by the existence of separate bands having either some strong bonding character or mostly pure atomic character [13]. It is then natural to adopt for such systems an idealized density of states per atom such as that pictured in figure 5 . It consists of $Z / 2$ bonding and antibonding states per spin direction and per atom and of $4-Z$ non bonding (or atomic like) states at $E=0$. We consider that $\beta$ is of the form $\beta^{0} \exp (-q R)$ as usual and take $\beta^{0}$ to be independent of the structure. We can thus calculate the total energy per atom $E_{\mathrm{T}}$ as a function of $N_{\mathrm{e}}$ and $Z$ and find the optimum value of $N_{\mathrm{e}}(Z)$ which minimizes $E_{\mathrm{T}}$. From figure 5 two possibilities have to be considered.

$3.1 N_{\mathrm{e}} \leqslant 8-Z$. - As the number of states in the two lower bands is $4-Z / 2$, the upper antibonding band is empty. On the other hand we have $N_{\mathrm{e}} \geqslant 4$ and we consider systems for which $Z \leqslant 4$, in which case the lower band is filled. The total energy per atom $E_{\mathrm{T}}$ can then be written

$E_{\mathrm{T}}=-Z \beta_{0} \exp (-q R)+\frac{Z}{2} C_{0} \exp (-p R)$.

After minimization with respect to $R$ this gives

$$
R_{\mathrm{e}}=\frac{1}{p-q} \ln \left(\frac{p C_{0}}{2 q \beta_{0}}\right)
$$

independent of $Z$ and a cohesive energy

$$
E_{\mathrm{c}}=Z \beta_{0}\left(1-\frac{q}{p}\right)\left(\frac{2 q \beta_{0}}{p C_{0}}\right)^{\frac{q}{p-q}}
$$

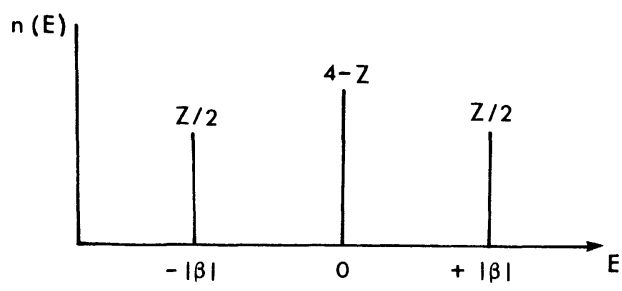

Fig. 5. - Simplified density of states for $Z<4$. directly proportional to $Z$. In this range the best value of $Z$ (i.e. which gives $E_{\mathrm{c}}$ maximum) is clearly given by $Z$ maximum equal to $8-N_{\mathrm{e}}$.

$3.2 N_{\mathrm{e}} \geqslant 8-Z$. - Now the upper antibonding band is partially filled with $\left(N_{\mathrm{e}}-8+Z\right)$ electrons. This cancels part of the bonding energy of the $Z$ electrons in the lower band. The total energy per atom becomes

$$
E_{\mathrm{T}}=-\left(8-N_{\mathrm{e}}\right) \beta_{0} \exp (-q R)+\frac{Z}{2} C_{0} \exp (-p R)
$$

which after minimization leads to

$$
R_{\mathrm{e}}=\frac{1}{p-q} \ln \left(\frac{p C_{0}}{2 q \beta_{0}} \cdot \frac{Z}{8-N_{\mathrm{e}}}\right)
$$

and

$$
E_{\mathrm{c}}=\left(8-N_{\mathrm{e}}\right) \beta_{0}\left(1-\frac{q}{p}\right)\left(\frac{2 q \beta_{0}}{p C_{0}} \cdot \frac{8-N_{\mathrm{e}}}{Z}\right)^{\frac{q}{p-q}} .
$$

In that range, for fixed $N_{\mathrm{e}}, E_{\mathrm{c}}$ is maximum when $Z$ is minimum, i.e. again equal to $8-N_{\mathrm{e}}$. The net result of the whole calculation is that, given the value of $N_{\mathrm{e}}$, the most stable covalent structure for the system corresponds to a coordination number $Z$ such that

$$
Z=8-N_{\mathrm{e}} \text {. }
$$

This is the famous $8-N_{\mathrm{e}}$ or Grimm-Sommerfeld rule which has been used with great success for a classification of the structures of a large variety of covalent systems [7]. For that particular value of $Z, R_{\mathrm{e}}$ is clearly given by (13) i.e. is independent of $N_{\mathrm{e}}$, a fact which is largely consistent with the experimental trends as shown in figure 2 . As regards $E_{\mathrm{c}}$ it is given by (14) with $Z$ equal to $8-N_{\mathrm{e}}$ i.e. it decreases linearly. Both results are schematically given on figure 6 which is in strikingly good agreement with figure 1. This leads us to conclude that the essential physics are contained in our very simple picture. This conclusion will be confirmed by the following numerical calculation.

\section{Complete numerical calculation for different struc- tures.}

The fair success of the simplified models described above has led us to push the calculation much further to obtain quantitative predictions. We have thus performed a complete calculation of the three following quantities $E_{\mathrm{c}}, R_{\mathrm{e}}, R_{\mathrm{e}}^{2}\left(\frac{\mathrm{d}^{2} E_{\mathrm{c}}}{\mathrm{d} R^{2}}\right)_{R_{\mathrm{e}}}$ for different possible structures. We have done this for the f.c.c., b.c.c., simple cubic and diamond crystalline structures. For systems with $Z<4$ we have used a molecular type of description plus perturbation theory to account for the 
corrective terms. All these calculations have been made using a nearest-neighbour tight binding approximation except for the b.c.c. lattice where the second nearest neighbours have been included since they play an important rôle.

To be more specific we have first made detailed calculations for the silicon row of the periodic table. All parameters, i.e. the $\beta_{i j}^{0}, C_{0}, p, q$ have been determined from a fit to known physical properties of silicon. The $\beta_{i j}^{0} \exp \left(-q R_{\mathrm{e}}\right)$ are deduced from the band structure while $C_{0}, p$ and $q$ are then calculated in such a way that the model gives the correct value for the cohesive energy $E_{\mathrm{c}}$, the equilibrium distance $R_{\mathrm{e}}$ and compressibility $B$. This leads to the values reported in table I. From these values it is then a simple matter to calculate the sum of one-electron energies for different structures and different values of $N_{\mathrm{e}}$. We do this by taking $E_{\mathrm{p}}$ as the origin of energies $\left(E_{\mathrm{A}}\right.$ is then only a function of $E_{\mathrm{s}}$ equal to $\left.\left(E_{\mathrm{s}}-E_{\mathrm{p}}\right)\right)$. This can
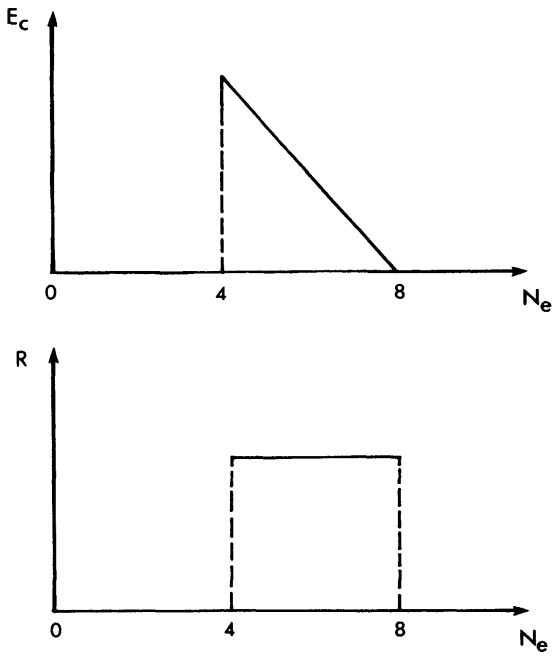

Fig. 6. - Trends in $E_{\mathrm{c}}$ and $R$ versus $N_{\mathrm{e}}$ predicted by the simplified model of Fig. 5.

Table I. - Values of the tight binding parameters obtained from a fit to the band structure of silicium. $R_{\mathrm{Si}}$ is the equilibrium distance in this material. The $\beta_{i j}$ are equal to $\beta_{i j}^{0} \exp \left(-q R_{\mathrm{Si}}\right)$.

\begin{tabular}{|c|c|c|c|c|c|c|}
\hline$\beta_{\mathrm{ss}}$ & $\beta_{\mathrm{s} \sigma}$ & $\beta_{\sigma \sigma}$ & $\beta_{\pi \pi}$ & $C_{0} \exp \left(-p R_{\mathrm{Si}}\right)$ & $p R_{\mathrm{Si}}$ & $q R_{\mathrm{Si}}$ \\
\hline$-1.93 \mathrm{eV}$ & $-2.23 \mathrm{eV}$ & $-3.22 \mathrm{eV}$ & $-0.83 \mathrm{eV}$ & $4.72 \mathrm{eV}$ & 4.66 & 2.99 \\
\hline
\end{tabular}

be done for different values of the nearest neighbour distance $R$. The equilibrium distance $R_{\mathrm{e}}$ will be the value which minimizes the quantity

$$
E_{\mathrm{T}}=E_{\mathrm{A}}+\frac{Z}{2} C_{0} \exp (-p R)
$$

while the cohesive energy will be given by

$$
E_{\mathrm{c}}=N_{\mathrm{s}} E_{\mathrm{s}}-E_{\mathrm{A}}-\frac{Z}{2} C_{0} \exp \left(-p R_{\mathrm{e}}\right)
$$

$N_{\mathrm{s}}$ being the number of $\mathrm{s}$ electrons in the free atom. We have performed the calculations of $R_{\mathrm{e}}$ and $E_{\mathrm{c}}$ numerically for the f.c.c., b.c.c., simple cubic and diamond structures. The results for $E_{\mathrm{c}}$ are given on figure 7 and will be discussed later. The cases for which $Z<4$ are more difficult to handle in view of the complexity of the corresponding crystalline structures. However we shall make use of the strong covalent character of the corresponding systems to devise methods for calculating $E_{\mathrm{c}}$ and $R_{\mathrm{e}}$. For this we consider the local situations of figure 8 describing the cases $Z=3,2,1$ and where we assume, to simplify, that bonding directions make angles equal to $90^{\circ}$. The electronic structure can then be obtained simply by starting from a molecular-like model and correcting it by second order perturbation theory. The molecular model can be obtained by considering for each atom $Z$ p states pointing towards its nearest-

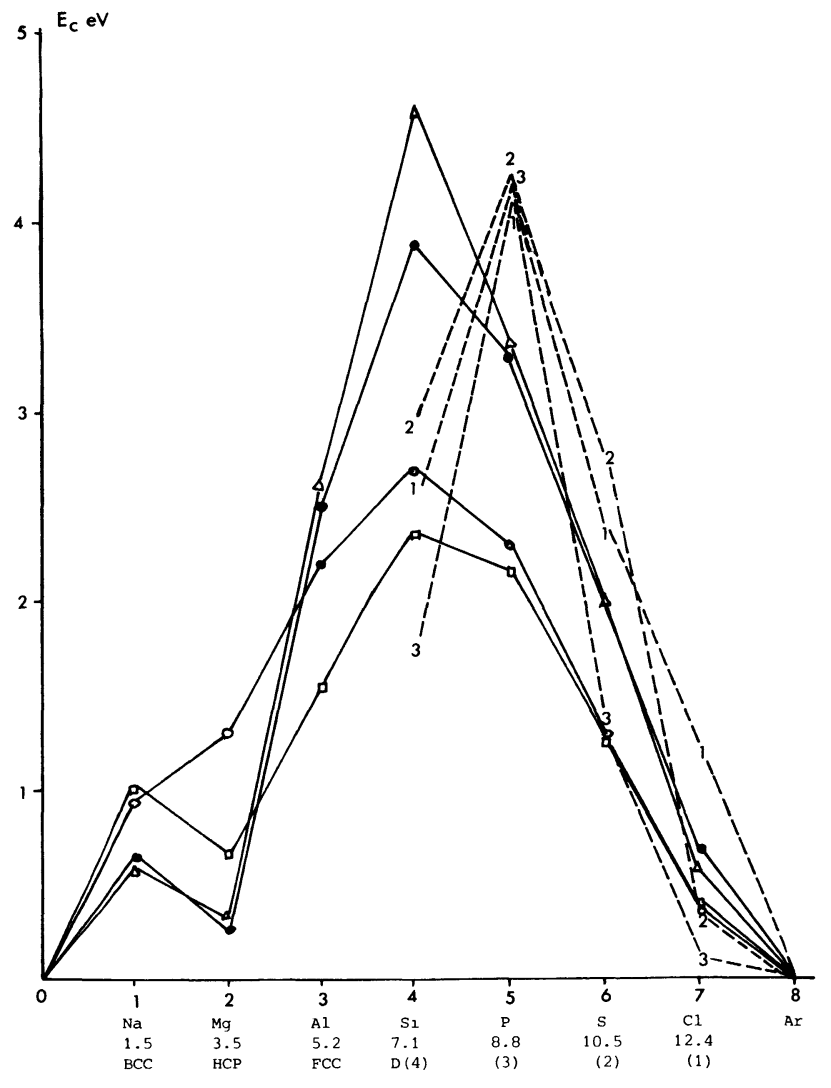

Fig. 7. - Calculated values of $E_{\mathrm{c}}$ for seven different structures : $(\bullet)$ simple cubic, $(\triangle)$ diamond, $(O)$ f.c.c., $(\square)$ b.c.c., and finally in dashed lines, structures with $Z=1,2$ and 3. 


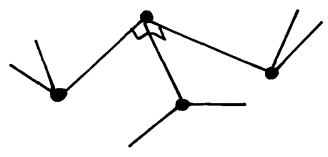

(a)

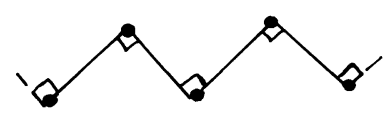

(b)

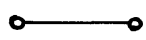

(c)

Fig. 8. - Local bonding for structures with $Z=1,2$ and 3.

neighbours (these will be the bonding $\mathrm{p}$ states) and $3-Z$ orthogonal (or non bonding) $p$ states. The bonding $\mathrm{p}$ states will be involved in $\sigma$ bonds each one giving rise to a bonding and an antibonding level at $\pm \beta_{\sigma \sigma}$. At that level of approximation we neglect interactions between non bonding $p$ states which thus give rise to a flat band at $E_{\mathrm{p}}$. Finally the difference $E_{\mathrm{p}}-E_{\mathrm{s}}$ is taken to be large enough so that the s states also remain atomic like.

In this " molecular model » the density of states is that given on figure 9 . As $N_{\mathrm{e}}>4$ the $\mathrm{s}$ band is always filled as in the free atom and does not contribute to the bonding. The remaining part of the density of states then becomes exactly identical to that of figure 5 used in section 3 with $\beta^{0}$ given by $\beta_{\sigma \sigma}^{0}$. However this model is oversimplified and further interactions have to be included. Some of them broaden the flat bands, others giving rise to shifts of these bands. We have determined the contribution of these corrective terms to $E_{\mathrm{c}}$ and $R_{\mathrm{e}}$, essentially through the use of perturbation theory. This is detailed in the appendix.

\section{Discussion.}

Figure 7 describes the results for $E_{\mathrm{c}}$ corresponding to the seven different structures considered. The atomic values for $E_{\mathrm{p}}-E_{\mathrm{s}}$ are taken from reference 14 except for $\mathrm{Na}$ and $\mathrm{Mg}$ where they have been deduced by simple extrapolation of the straight line formed by the other values (for $\mathrm{Na}$ there is no quoted value for $E_{\mathrm{p}}$ and for $\mathrm{Mg}$ the result is extremely sensitive to $E_{\mathrm{p}}-E_{\mathrm{s}}$ whose value differs for different authors). On this figure are also indicated the observed

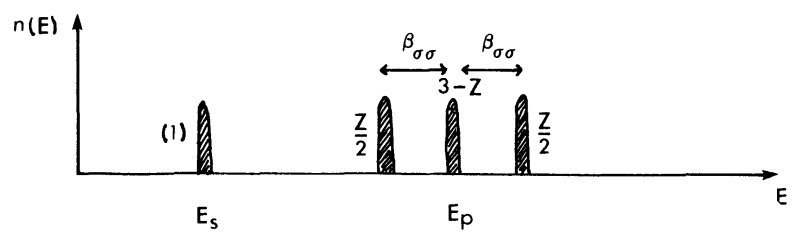

Fig. 9. - Density of states in the molecular model of systems with $Z=1,2$ and 3. structures for $N_{\mathrm{e}} \leqslant 4$ and the coordination number for $N_{\mathrm{e}} \geqslant 4$. On the whole it is clear that our tight binding description does quite a good job. The b.c.c. structure of $\mathrm{Na}$ is correctly predicted but with almost identical energy as the f.c.c. structure (the order can be reversed by changing $\left.E_{\mathrm{p}}-E_{\mathrm{s}}\right)$. For $N_{\mathrm{e}}=2(\mathrm{Mg})$ the compact structure $(Z=12)$ is clearly favoured as obtained experimentally (h.c.p.). It is only for $\mathrm{Al}$ that incorrect ordering is obtained, the diamond and simple cubic structures being more stable than the observed f.c.c. structure. In all remaining cases with $Z \leqslant 4$, essentially correct results are obtained confirming the $8-N_{\mathrm{e}}$ rule. An interesting point is that, for $N_{\mathrm{e}}=5$, all three structures with $Z=1,2,3$ give practically identical results for $E_{\mathrm{c}}$. This is perhaps partly due to our simplified description of their structure but is also probably related to the known occurrence in such systems of changes in local coordination (the valence alternation pairs [15]).

In figure 10, we give the theoretical curve of $E_{\mathrm{c}}$
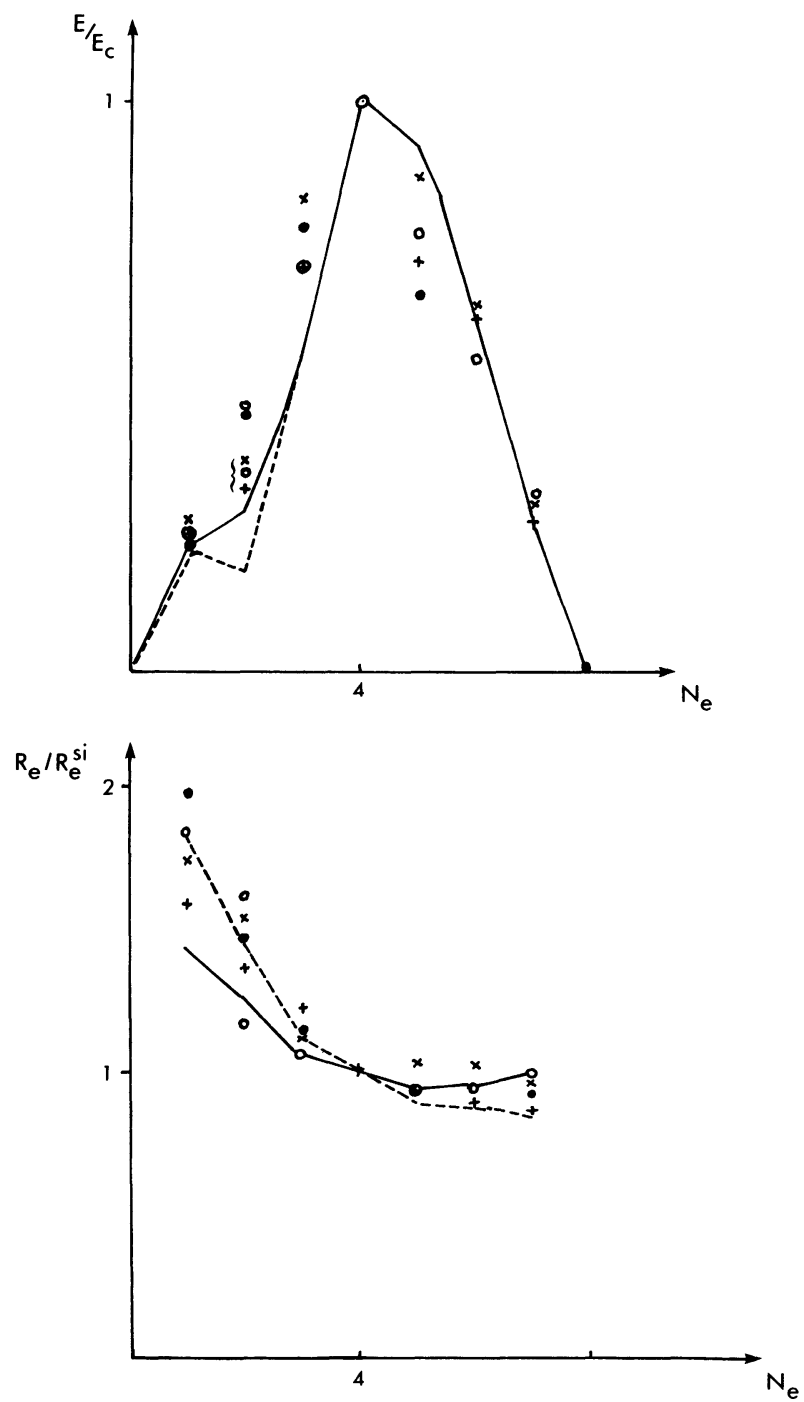

Fig. 10. - Trends in the ratio of $E_{\mathrm{c}}$ and $R_{\mathrm{e}}$ to their values for the corresponding column IV element. The points are experimental values; the continuous or dashed lines represent our theoretical results. 
and $R_{\mathrm{e}}$ versus $N_{\mathrm{e}}$. For this we have plotted the ratios of these quantities to their values for silicon, i.e. $N_{\mathrm{e}}=4$. In the same figure we give the experimental points, not only for the silicon row but also for the three other rows containing $\mathrm{C}, \mathrm{Ge}$ and $\mathrm{Sn}$. It appears clearly that the experimental points almost follow a unique curve which is quite well reproduced by the theoretical one (note that we have not tried to optimize the values of $E_{\mathrm{p}}-E_{\mathrm{s}}$ to get a better theoretical curve). The most important discrepancies for $E_{\mathrm{c}}$ concern the values $N_{\mathrm{e}}=3$ and 5 . For $N_{\mathrm{e}}=3$ it could easily be corrected by a slight change in $E_{\mathrm{p}}-E_{\mathrm{s}}$. This is not true for $N_{\mathrm{e}}=5$ where the theoretical value is now in excess. This might be due to our oversimplified description of the structure where we have in fact maximized the cohesion by neglecting more distant interactions present in the actual structure.

The predicted trend for $R_{\mathrm{e}}$ in figure 10 is again correct but the relative variation is not important enough. The natural explanation for this comes from the fact that, along a given row, the atomic orbitals become more and more compact as $N_{\mathrm{e}}$ increases. This means that $p$ and $q$ increase with $N_{\mathrm{e}}$ along the row. The simplest model to account for this is to consider that the ratio $p / q$ remains constant (which can be roughly justified in an atomic orbital picture) and that the quantities $p R_{\mathrm{e}}$ and $q R_{\mathrm{e}}$ remain constant too. This is a completely coherent hypothesis since $R_{\mathrm{e}}$ is given by minimization of equation 19 with respect to $R$. As $E_{\mathrm{A}}$ depends on $R$ through the $\beta_{i j}$ we can write :

$0=\sum_{\beta_{i j}}\left(\frac{\partial E_{\mathrm{A}}}{\partial \beta_{i j}}\right)_{R_{\mathrm{e}}}\left(-q \beta_{i j}^{0} \mathrm{e}^{-q R_{\mathrm{e}}}\right)-p \frac{Z}{2} C_{0} \mathrm{e}^{-p R_{\mathrm{e}}}$

leading to

$$
(p-q) R_{\mathrm{e}}=\ln \left[-\frac{p}{2 q} \frac{Z C_{0}}{\sum_{\beta_{i j}}\left(\frac{\partial E_{\mathrm{A}}}{\partial \beta_{i j}}\right)_{R_{\mathrm{e}}}}\right]
$$

If $p / q$ and $p R_{\mathrm{e}}$ remain constant, the term on the right remains unchanged showing that $(p-q) R_{\mathrm{e}}$ is also constant, showing that the assumption is completely consistent. We take for $(p-q)$ a linear expansion around its value for $N_{\mathrm{e}}=4$ given by

$$
(p-q)=(p-q)_{N_{\mathrm{e}}=4}\left[1+a\left(N_{\mathrm{e}}-4\right)\right],
$$

$a$ being a positive constant. The result for $R_{\mathrm{e}}$ can then be expressed as

$$
R_{\mathrm{e}}=\frac{\left(R_{\mathrm{e}}\right)_{0}}{1+a\left(N_{\mathrm{e}}-4\right)}
$$

where $\left(R_{\mathrm{e}}\right)_{0}$ are the values obtained before, assuming $p-q$ to be a constant equal to $(p-q)_{N_{\mathrm{e}}=4}$. The extra parameter to determine is $a$ for which we take the value 0.067 . This gives the corrected curve indicated by a dashed line on figure 10 in much better agreement with the experimental situation at least for $N_{\mathrm{e}}<4$. An intermediate value of $a$ would improve the agreement for $N_{\mathrm{e}}>4$ and only slightly destroy it for $N_{\mathrm{e}}<4$ and might thus be more appropriate.

Let us now discuss the bulk modulus $B$. From our point of view this is not a very convenient quantity and it is better to consider the product $B \Omega$ where $\Omega$ is the atomic volume. One can easily show that $B \Omega$ is equal to :

$$
B \Omega=\frac{R_{\mathrm{e}}^{2}}{9} \frac{\mathrm{d}^{2} E_{\mathrm{T}}}{\mathrm{d} R_{\mathrm{e}}^{2}}
$$

where $E_{\mathrm{T}}$ is the total energy per atom defined above. As before, we have made the theoretical calculation only for the silicon row and the results are given on figure 11 in dimensionless units (where $B \Omega$ is divided by its value for $N_{\mathrm{e}}=4$ ). The corresponding experimental points are also given on this figure and again one can see that the overall trend is correctly predicted. The order of magnitude of $B \Omega$ is correctly obtained in view of the fact that we have not tried to modify the parameters to obtain better agreement from case to case. In general for $N_{\mathrm{e}}<4, B \Omega$ is overestimated the theoretical value for $N_{\mathrm{e}}=2$ leading to maximum error (this is not surprising since in this case the results are strongly sensitive to $E_{\mathrm{p}}-E_{\mathrm{s}}$ ).

Finally it is worth mentioning that the parameters $\beta_{\alpha \beta}$ which have been shown to work for the cohesive properties have the correct order of magnitude as regards the corresponding band structure of the elements. They were obtained by a fit in the case of $\mathrm{Si}$. If we consider $\mathrm{Na}$, a free-electron approximation would give a distance between the Fermi energy and the bottom of the band equal to $5.7 / R_{\mathrm{e}}^{2}$ in atomic

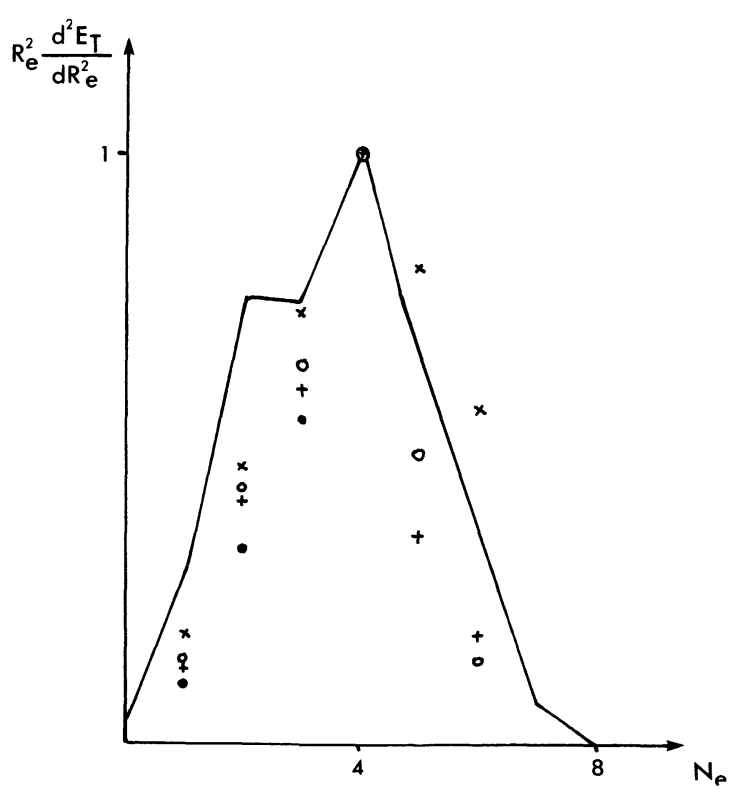

Fig. 11. - Trends in the reduced value of $R_{\mathrm{e}} \frac{\mathrm{d}^{2} E_{\mathrm{T}}}{\mathrm{d} R_{\mathrm{e}}^{2}}$ with the same conventions as in Fig. 10. 
units while in a nearest-neighbour tight binding treatment the same quantity is given by $8 \beta_{\mathrm{ss}}$. This would lead to $\beta_{\mathrm{ss}} \sim 0.17 \mathrm{eV}$ which is exactly the same value which would be obtained in our model using the expression $\beta_{\mathrm{ss}}^{0} \exp \left(-p R_{\mathrm{e}}\right)$ with the value of $R_{\mathrm{e}}$ obtained from the dashed line on figure 10 and $N_{\mathrm{e}}=1$. A similar agreement is found for $N_{\mathrm{e}}=6$, when using again $R_{\mathrm{e}}$ given by the dashed line of figure 10 and scaling the $\beta_{\alpha \beta}$ in the ratio of the cohesive energies of the corresponding columns IV element. This gives for Se and Te values of $\beta_{\sigma \sigma}$ equal to $4.08 \mathrm{eV}$ and $3.29 \mathrm{eV}$ which are again in good agreement with those obtained from a fit to the band structure of these materials [13].

\section{Conclusion.}

We have applied a tight binding treatment to the description of the trends in the cohesive properties of the sp elements. The values of the parameters have been obtained from a fit to silicon (band structure, cohesive energy, nearest neighbour distance and compressibility) and have then been applied to other elements of the same row. For other rows, simple scaling of the parameters for the atom of column IV has been used. We have shown that this method gives a quite good description of the trends, not only for the cohesive energy and nearest-neighbour distance but also for the compressibility. This shows that the cohesive properties are really related in a simple manner to the filling of the sp band. We have also calculated the relative stability of different structures and obtained fairly correct results except for the elements of column III. For more than four electrons per atom we have been able to demonstrate analytically the Grimm-Sommerfeld rule (or $8-N_{\mathrm{e}}$ rule), a conclusion which was then confirmed by numerical calculation. Finally the parameters deduced by our method have been shown to be also valid for a description of the band structure of the elements.

\section{Appendix.}

BAND STRUCTURE ENERGY FOR $N_{\mathrm{e}}>4$. - We want here to determine the attractive part $E_{\mathrm{A}}$ of the energy per atom not only versus $N_{\mathrm{e}}$ but also for different coordination numbers $Z$, i.e. $E_{\mathrm{A}}\left(Z, N_{\mathrm{e}}\right)$. We start from the molecular description of figure 9 where the density of states is built up from flat bands. In this limit it is easy to calculate $E_{\mathrm{A}}$ which is essentially given by an integer times $-\left|\beta_{\sigma \sigma}\right|$. However more accurate values are needed and we must include for this the shift of these bands due to the coupling between molecular states belonging to different bands. We do this by second-order perturbation theory (except in one case which we discuss later). The only band which has no shift in our model is the lone pair band at $E_{\mathrm{p}}$. If we call $\Delta E_{\mathrm{s}}, \Delta E_{\mathrm{B}}, \Delta E_{\mathrm{A}}$ the shifts of the s, bonding and antibonding bands respectively it is easy to show that (taking $E_{\mathrm{p}}=0$ and $\beta_{\sigma \sigma}<0$ )

$$
\begin{aligned}
\Delta E_{\mathrm{s}} & =\frac{Z}{2} \beta_{\mathrm{s}}^{2}\left(\frac{1}{E_{\mathrm{s}}-\beta_{\sigma \sigma}}+\frac{1}{E_{\mathrm{s}}+\beta_{\sigma \sigma}}\right) \\
\Delta E_{\mathrm{B}} & =\frac{\beta_{\mathrm{s} \sigma}^{2}}{\beta_{\sigma \sigma}-E_{\mathrm{s}}}+\alpha \frac{\beta_{\pi \pi}^{2}}{\beta_{\sigma \sigma}} \\
E_{\mathrm{A}} & =-\frac{\beta_{\mathrm{s} \sigma}^{2}}{\beta_{\sigma \sigma}+E_{\mathrm{s}}}-\alpha \frac{\beta_{\pi \pi}^{2}}{\beta_{\sigma \sigma}}
\end{aligned}
$$

where $\alpha=1 / 2$ for $Z=3,1 / 4$ for $Z=2,0$ for $Z=1$. A final contribution to $E_{\mathrm{A}}$ comes from the broadening of the lone pair band when it is incompletely filled. There is no such contribution for $Z=3$. For $Z=2$ one has a $\pi$ bonded chain with one state per atom whose contribution to $E_{\mathrm{A}}$ gives $\frac{4}{\pi} \beta_{\pi \pi}$ when half filled. Finally for $Z=1$ one can form two bonding $\pi$ states at energy $\beta_{\pi \pi}$ and two antibonding $\pi$ states at $-\beta_{\pi \pi}$ whose contribution to $E_{\mathrm{A}}$ depends upon their filling. From the considerations given above it is now easy to obtain $E_{\mathrm{A}}\left(Z, N_{\mathrm{e}}\right)$ for each couple $Z$ and $N_{\mathrm{e}}$ by filling the lower bands with the available electrons.
[1] Values of the cohesive energies have been taken from : a) KITTEL, C., Introduction to Solid State Physics (J. Wiley and Sons Inc. New York, London, Sydney) 1967.

b) Pauling, L., Nature of the Chemical Bond (Cornell University Press, New York) 1948.

[2] Nearest neighbour distances have been taken from Slater, J. C., Quantum Theory of Molecules and Solids, vol. 2 (Mc Graw-Hill Book Company) 1965, p. 307.

[3] For the compressibilities see reference $1 a$ and also : a) MotT, N. F. and Jones, H., The theory of the properties of metals and alloys (Dover Inc.) 1958.

b) Gschneider, K. A., Solid State Physics, F. Seitz and D. Turnbull eds., vol. 16 (Academic Press, New York) 1975, p. 275.

[4] Friedel, J., in « The Physics of Metals » ed. J. M. Ziman (Cambridge University Press) 1969, p. 340.

[5] Ducastelle, F., J. Physique 31 (1970) 1055.

[6] Friedel, J., J. Physique 39 (1978) 651.

[7] PARThe, E., Cristallochimie des structures tétraédriques (ed. Gordon and Breach) 1972. 
[8] Yin, M. T. and Cohen, M. L., Phys. Rev. Lett. 45 (1980) 1004.

[9] Slater, J. C. and Koster, G. F., Phys. Rev. 94 (1954) 1498.

[10] Harrisson, W. A., The Physics of Solid State Chemistry, Festkörperprobleme XVII, Springer Tracts (Springer Verlag, Berlin) 1977.

[11] Lannoo, M., J. Physique 40 (1979) 461.

[12] LoHEZ, D. and LANNOO, M., to be published. HARRISSON, W. A., to be published.
[13] Bensoussan, M. and Lannoo, M., J. Physique 40 (1979) 749.

[14] For the free atom values $E_{\mathrm{s}}$ and $E_{\mathrm{p}}$ see Slater J. C., Quantum Theory of Atomic Structures, vol. 1 (Mc Graw-Hill, New York) 1960, p. 206 ; or

HARrisson, W. A., Electronic Structure and the Properties of Solids, The Physics of the Chemical Bond (Freeman, New York) 1980, chap. 2.

[15] Kastner, M., Adler, D. and Fritzche, H., Phys. Rev. Lett. 37 (1976) 1504. 